\title{
Gastronomía en valor: un estudio de caso de la horchata y los fartons en Valencia (España)*
}

\author{
[Versión en Castellano]

\begin{abstract}
Gastronomy in Value: a Case Study of Horchata and Fartons in Valencia (Spain)

Gastronomia em valor: um estudo de caso da horchata e fartons em Valencia (Espanha)

Valencia (Espanha)
\end{abstract}

Recibido 20 de abril, 2019 Aceptado 25 de octubre, 2019.

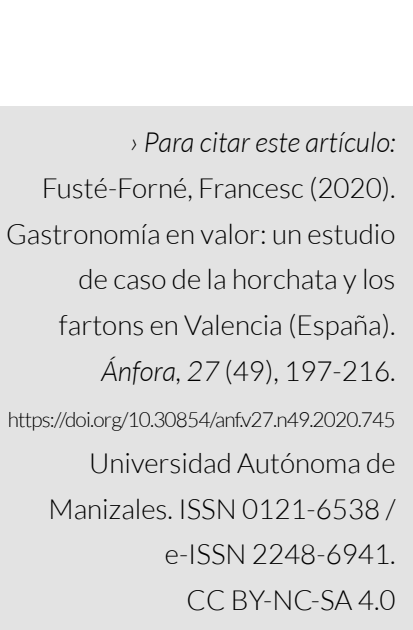

CC BY-NC-SA 4.0

Francesc Fusté-Forné** https://orcid.org/0000-0002-3800-9284

España

\section{Resumen}

Objetivo: aproximar la geografía local de dos productos característicos de la gastronomía valenciana, esto es, la horchata y los fartons. Metodología: para ello, se utilizó la observación no participante como fuente de información primaria y la bibliografía académica y sectorial como fuente secundaria. Resultados: se advirtió un elevado grado de identificación de estos dos productos con la cultura y la gastronomía valencianas y, por lo tanto, de su capacidad de transmisión de una identidad auténtica. Conclusiones: la proyección y comercialización de las prácticas y experiencias asociadas a los productos locales ayudan, también, a hacer frente a las tendencias globalizadoras del contexto gastronómico actual.

\footnotetext{
* Artículo derivado de la Investigación titulada "Gastronomía en valor: un estudio de caso de la horchata y los fartons en Valencia (España)". La financiación del proyecto fue asumida por el investigador, quien declara que no hubo conflicto de interés en la ejecución del proyecto de investigación.

** Doctor en Turismo, Derecho y Empresa. Profesor Asociado en el Departamento de Empresa, Facultad de Turismo, Universitat de Girona. Plaça Josep Ferrater i Móra 1, 17004, Girona, Catalunya, España. Correo: francesc.fusteforne@udg.edu; researchexperiencetourism@gmail.com
} 
Palabras-clave: Desenvolvimento local; Turismo gastronômico; Cozinha Regional de Valência, Espanha; Ecoturismo; Horchata; Fartons.

\section{Abstract}

Objective: to approximate the local geography of two characteristic products of Valencian gastronomy, that is, Horchata and Fartons. Methodology: non-participant observation was used as the primary source of information. The academic and sectoral bibliography was implemented as the secondary source. Results: a high degree of identification of these two products with Valencian culture and gastronomy and, therefore, their ability to transmit an authentic identity were noted. Conclusions: the projection and commercialization of practices and experiences associated with local products also help to face globalizing trends in the current gastronomic context.

Keywords: Local development; Gastronomic tourism; Regional Cuisine of Valencia, Spain; Ecotourism; Horchata; Fartons.

\section{Resumo}

Objetivo: aproximar ageografialocal de dois produtos característicos dagastronomia valenciana, isto é, horchata e fartons. Metodologia: para isso, a observação não participante foi usada como fonte primária de informação e a bibliografia acadêmica e setorial como fonte secundária. Resultados: houve um alto grau de identificação desses dois produtos com a cultura e a gastronomia valenciana e, portanto, sua capacidade de transmitir uma identidade autêntica. Conclusões: a projeção e comercialização de práticas e experiências associadas a produtos locais também ajudam a enfrentar as tendências globalizantes no atual contexto gastronômico.

Palavras-chave: Desenvolvimento local; Turismo gastronômico; Cozinha Regional de Valência, Espanha; Ecoturismo; Horchata; Fartons. 


\section{Introducción}

La alimentación, la cocina y la gastronomía se encuentran entre los elementos más trascendentales de cualquier cultura. Son varios los autores que desarrollan el papel de la gastronomía como una de las características fundacionales de la cultura (Berno, 2017; Fischler, 1988; Sims, 2009). En este sentido, la identidad se transmite a través de la gastronomía, que ofrece una muestra genuina de las geografías culturales y naturales de un lugar (Tresserras y Medina, 2008). Aquí, la horchata y los fartons son un ejemplo de la cultura gastronómica valenciana, no solo en el ámbito nacional sino también en el contexto internacional, estrechamente ligados a los valores territoriales y sociales de la alimentación y la cocina regional.

El objetivo de este artículo es analizar la horchata y los fartons como productos típicos valencianos, y discutir su potencial como elementos de atracción turística. En particular, este artículo desarrolla estos dos productos desde su importancia como parte de la oferta gastronómica de Valencia, muestra de su identidad cultural y natural. Después de contextualizar el marco teórico y definir la metodología utilizada, el artículo analiza la geografía de la horchata y los fartons para finalmente apuntar, en la sección de conclusiones, las limitaciones, implicaciones y oportunidades de investigación futura alrededor de esta temática.

\section{Contexto teórico}

La descubierta de la cultura a partir de los paisajes gastronómicos es uno de los puntales del turismo gastronómico. Así, los paisajes gastronómicos se entienden, de acuerdo con Adema (2006), como "los paisajes sociales, culturales, políticos, económicos o históricos que, de una u otra forma, tienen que ver con la comida" (p. 13). De este modo, la gastronomía es uno de los elementos fundacionales de cualquier cultura, la cual se entiende como el conjunto de todos los elementos que conforman la identidad de un pueblo, incluyendo la suma de los paisajes culturales y naturales, que expresan un sentido de lugar único asociado a cada identidad (Antrop, 2005; Smith, 2015; Tellström, Gustafsson y Mossberg, 2006) y a cada contex to geográfico.

Entre estos paisajes, los gastronómicos no son únicamente manifestaciones del patrimonio culinario, sino que emergen como asociaciones intangibles entre un lugar determinado y su comida (Fusté-Forné, 2016b). En este contexto, el reconocimiento de la UNESCO del patrimonio gastronómico como bien inmaterial se produjo en el 2010, lo cual es un ejemplo de la importancia de la gastronomía, tanto en relación con la preservación de los paisajes locales, como en cuanto a su proyección turística. 
El turismo gastronómico se refiere al desplazamiento a un lugar con la finalidad no solo de probar productos y platos, visitar centros productores o puntos de venta, asistir a ferias y festivales o cualquier otra actividad turística basada en la gastronomía (Hall y Sharples, 2003), sino también para conocer los sentidos y significados asociados a la gastronomía y la cultura y a las tradiciones culinarias. Esta definición incluye una gran variedad de oportunidades que se generan alrededor del turismo gastronómico, que tiene entre sus principales objetivos el desarrollo económico local.

De este modo, en la actual literatura académica entorno a las prácticas del turismo gastronómico se destacan los productos locales como uno de sus principales pilares, que a la vez son claves para este desarrollo regional (Bessière, 1998; Díaz y Llurdés, 2013). Así, aunque el vino es el que se ha desarrollado más ampliamente (Hall, Sharples, Cambourne y Macionis, 2009; López-Guzmán, García y Rodríguez, 2013) otros productos, tanto de mar como de montaña, tales como el aceite (Guerra, Moreno y López, 2014), los mejillones (Lund, 2015) o el queso (Fusté-Forné, 2016a), para citar solo algunos ejemplos, también se han estudiado desde el punto de vista de la autenticidad, el desarrollo regional y la experiencia turística.

Además, hay platos que también han sido tratados en trabajos científicos alineados con los estudios culturales y turísticos, como es el caso de la paella (Duhart y Medina, 2008) u otros ejemplos internacionales como los fideos japoneses (Kim y Ellis, 2015). Todos estos elementos culinarios y gastronómicos, símbolos de una identidad local, son así mismo utilizados para la promoción y el marketing de destinos (Cohen y Avieli, 2004; Hjalager y Richards, 2002).

En el contexto de globalización actual es importante hacer también hincapié en el conflicto local-global. Por un lado, la diversidad geográfica es el origen de la diferenciación culinaria regional y el punto de partida para su aprovechamiento turístico (Cànoves y Villarino, 2000). No obstante, en determinadas ocasiones, los turistas prefieren aquellos productos con los que ya se encuentran familiarizados (Belisle, 1983), lo cual confiere una tranquilidad y seguridad en términos económicos y socioculturales. Cualquiera que haya consumido en una cadena de comida rápida sabe qué ofrece, qué sabor tiene, y cuánto cuesta, independientemente de su localización. Esto beneficia los procesos de globalización vinculados a la homogeneización de la gastronomía como ingrediente cultural.

Ante esto, la autenticidad local es una manifestación de los paisajes culturales y naturales (Hughes, 1995), y puede expresarse mediante la gastronomía. Cuando se consume un producto local no solo se incorporan las cualidades nutricionales del alimento, sino también unas cualidades simbólicas, relacionadas con la apropiación efímera de la identidad local (Bessière, 1998), así como sus significados históricos y socioculturales que en el proceso de intercambio entre 
anfitriones e invitados son parte de un ritual asociado a la hospitalidad y al respeto al otro (Korstanje, 2010). Por lo tanto, la valorización y promoción de la cultura gastronómica local representa un importante factor de atracción turística (Fusté-Forné, 2019; Hernández-Rojas y Dancausa-Millán, 2018; Leal-Londoño, Vázquez-Medina, Medina, 2018).

En este sentido, los destinos gastronómicos deben ser capaces de ofrecer productos y experiencias que comuniquen de forma eficaz el vínculo entre la gastronomía, el territorio y las comunidades locales (Hillel, Belhassen, Shani, 2013). Esto tiene como finalidad que el turista se exponga pues a una auténtica experiencia culinaria - la gastronomía como una identidad cultural en un contexto geográfico particular (Fusté-Forné, 2015). En este caso, esto se analiza a partir de la relevancia histórica y sociocultural de la horchata y los fartons en Valencia, y su integración en el paisaje de la ciudad como fuente de atracción del turismo gastronómico.

\section{Metodología}

Para responder al objetivo de este estudio, se ha realizado una aproximación cualitativa, basada en un trabajo de observación de campo no participante (Somekh y Lewin, 2005), complementado con datos obtenidos mediante fuentes de información secundarias. En el ámbito del turismo, según la Organización Mundial del Turismo (2001), la investigación cualitativa es una estrategia usada con una finalidad descriptiva alrededor de un fenómeno de interés. En concreto, la observación de campo es el procedimiento de mirar detenidamente el objetivo de observación, y puede estar relacionada con 'monumentos' de diversa índole (Pardinas, 1969).

En este caso, el objetivo de la observación es recopilar la presencia de la horchata y los fartons en la geografía de Valencia, escogida debido a su capitalidad de la región de la Comunidad Valenciana. El trabajo de campo se ha realizado en el mes de mayo de 2018 en las calles céntricas de la ciudad de Valencia, tomando como ejes geográficos principales la Plaza del Ayuntamiento, el Mercado Central, y la estación ferroviaria Estació del Nord. La interpretación de los datos secundarios se ha basado en fuentes que permitan trazar el contexto histórico de la horchata y los fartons, tanto desde el punto de vista académico como sectorial. 


\section{Resultados}

Tanto la horchata como los fartons son productos que se pueden ver ampliamente representados en el día a día de la Comunidad Valenciana, y, en particular, de la ciudad de Valencia. Así, el paisaje gastronómico y cultural valenciano contiene abundantes referencias a la horchata, a los fartons, y a la horchata con fartons (por ejemplo, Figura 1).

Primero, cabe destacar que la horchata es una bebida que se elabora a partir de la chufa (Figura 2). El cultivo de las chufas se remonta a miles de años atrás, encontrando evidencias de su existencia por ejemplo en el Antiguo Egipto (Maroto, 1998). En este sentido,

La chufa ya se consumía en el Antiguo Egipto, así lo atestiguan las vasijas encontradas como parte del ajuar funerario de faraones, nobles y artesanos. Los egipcios cocían los tubérculos y los consumían como postre. Las sociedades persas y árabes utilizaron las chufas con fines terapéuticos, básicamente por sus propiedades digestivas y desinfectantes (Sirvent-Barcelona, 2018, p. 1).

Así, también hay antiguas referencias a persas y árabes, o al imperio chino (Consell Regulador D.O. Xufa de València, 2018; Maroto, 1998; Serrallach, 1927). Pero

El cultivo de la chufa llegó a la Huerta del Norte de Valencia, en el siglo XIII, de la mano de los árabes quienes trasladaron el cultivo desde el Sudán por hallar en el clima mediterráneo de Valencia unas condiciones idóneas para su plantación: un terreno arenoso, un clima suave y un alto grado de humedad (Sirvent-Barcelona, 2018, p. 1).

Sus propiedades medicinales, unidas a su consumo como una bebida refrescante, la empezaron a hacer popular como "leche de chufas" durante el dominio islámico de la Península Ibérica (Pascual y Maroto, 1984). 
Fusté-Forné, Francesc (2020). Gastronomía en valor: un estudio de caso de la horchata y los fartons en Valencia (España). Ánfora, 27(49), 197-216. https://doi.org/10.30854/anf.v27.n49.2020.745

Figura 1. Integración del consumo de la horchata y los fartons en el paisaje gastronómico de la ciudad de Valencia

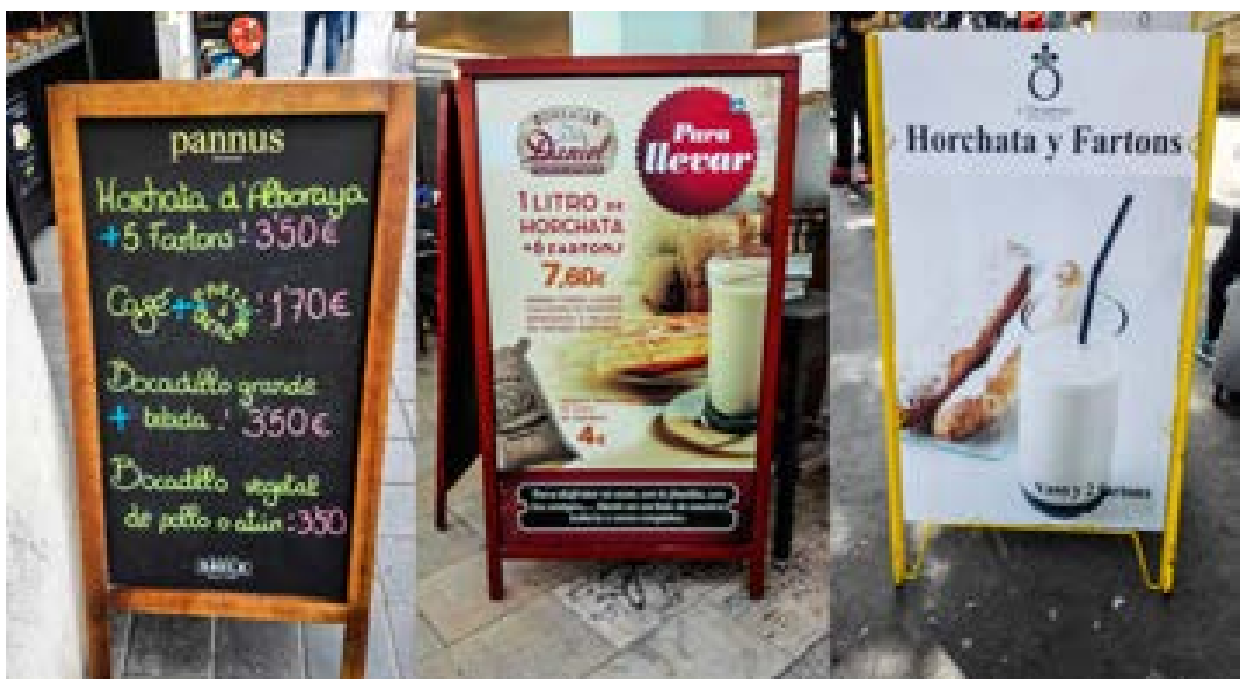

Fuente: elaboración propia

Figura 2. Productos y herramientas para la elaboración tradicional de la horchata de chufa. Valencia

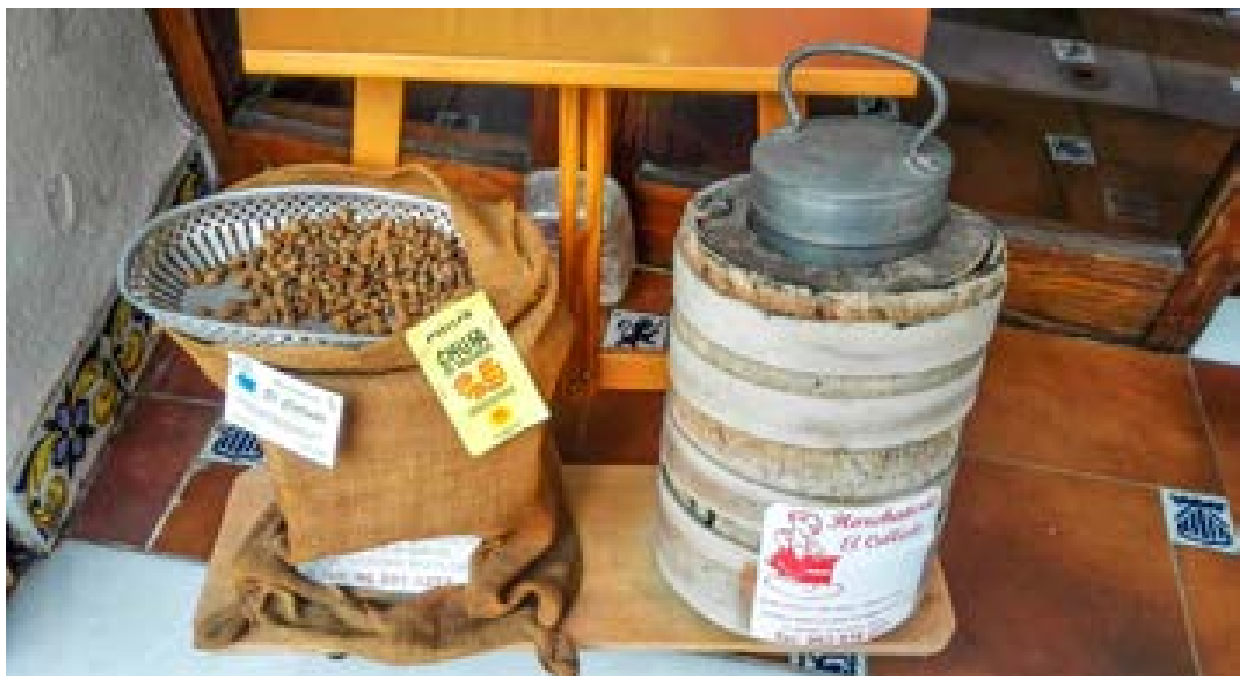

Fuente: elaboración propia 
De acuerdo con el Diari Oficial de la Comunitat Valenciana (2010), en el texto que aprueba el reglamento de la Denominación de Origen Protegida (DOP) Chufa de Valencia y el Consejo Regulador de la misma, se establece que

La producción de las chufas protegidas se realizará exclusivamente a partir de tubérculos de la variedad población de chufa (Cyperus esculentus L. var. sativus Boeck.) obtenidos o multiplicados, y cultivados en terrenos ubicados en los términos municipales siguientes: Albalat dels Sorells, Alboraya, Albuixech, Alfara del Patriarca, Almàssera, Bonrepòs i Mirambell, Burjassot, Foios, Godella, Meliana, Moncada, Paterna, Rocafort, Tavernes Blanques, València y Vinalesa (p. 21057).

Estos municipios se encuentran en la comarca valenciana de L'Horta Nord, siendo el único territorio de todo el Estado Español que cumple las condiciones climáticas y orográficas para el cultivo de la chufa.

La normativa regula las prácticas relacionadas con los procesos de cultivo, recolección, lavado y secado. En concreto, la chufa protegida se caracteriza por ser un tubérculo que puede "adquirir diversas formas, entre las que predominan las alargadas y las redondeadas, conocidas tradicionalmente como Llargueta y Ametlla, respectivamente" (Diari Oficial de la Comunitat Valenciana, 2010, p. 21057). Así mismo, "se distinguirán los siguientes tipos de chufa: a) Tierna: Se entiende por chufa tierna la recién recolectada y lavada. b) Seca: Se entiende por chufa seca el producto sometido a las operaciones de lavado y secado” (p. 21057).

A partir del cultivo de la chufa, pues, se llega a la elaboración de la horchata. De acuerdo con Álvarez, "en el territorio del antiguo Reino de Valencia se ingiere desde hace siglos [y] las primeras referencias escritas de la leche de chufas se remontan al siglo XIV” (Álvarez, 2017, p. 1). Otras referencias posteriores destacan el origen de esta bebida de la siguiente forma, también a modo de leyenda:

Desde el punto de vista etimológico, la palabra "horchata" procede del italiano y hace referencia en sus inicios al agua de cebada. El nombre deriva del participio italiano "orzata” y a su vez del latín "hordeata”, "hecha con orzo" (i.e. hordeum, voz latina que significa cebada). A pesar de ello, la leyenda cuenta que durante la Reconquista, el Rey de la Corona de Aragón, Jaume I “El Conquistador” tomó un vaso de horchata que le sirvió una muchacha. El Rey encantado con el sabor, se interesó por el nombre de la bebida. La chica respondió que era leche de chufa. El rey exclamó: "Això no és llet, això és OR, XATA!” ES DECIR “¡Esto no es leche, esto es oro, guapa!". Se originó un juego de palabras entre “or” y “xata”, dando el supuesto origen al vocablo valenciano "orxata", forma de escribirlo en dicho idioma (Sirvent-Barcelona, 2018, p. 2). 
En la actualidad, la horchata y su consumo se encuentran presentes no solo en las horchaterías (Figura 3), sino también en restaurantes o cafeterías (Figura 4) e incluso en puestos de comida móviles (Figura 5). Además, destaca también su presencia en los supermercados (Figura 6). Este último ejemplo tiene especial relevancia dada la creciente utilización de apartamentos por parte de visitantes y turistas, lo cual complementa su consumo turístico en restaurantes con la compra de productos en tiendas de alimentación y supermercados locales para cocinarlos en casa. Esto significa que su uso turístico está muy arraigado, tal y como lo demuestra una abundante y variada presencia de carteles que recurren a la horchata como principal reclamo -y también a los fartons-, con el objetivo de degustar la gastronomía local por parte de visitantes y turistas.

Figura 3. Ejemplo de puesto para el consumo de horchata (I). Valencia

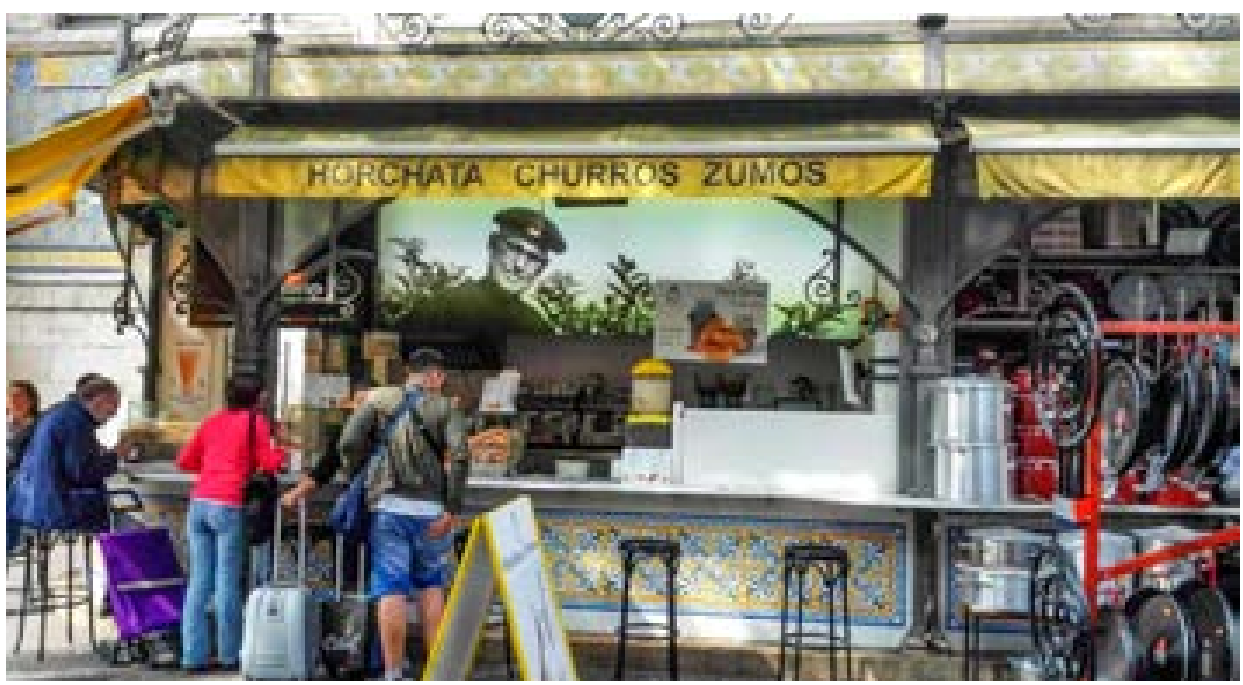

Fuente: elaboración propia 
Fusté-Forné, Francesc (2020). Gastronomía en valor: un estudio de caso de la horchata y los fartons en Valencia (España). Ánfora, 27(49), 197-216. https://doi.org/10.30854/anf.v27.n49.2020.745

Figura 4. Ejemplo de puesto para el consumo de horchata (II). Valencia

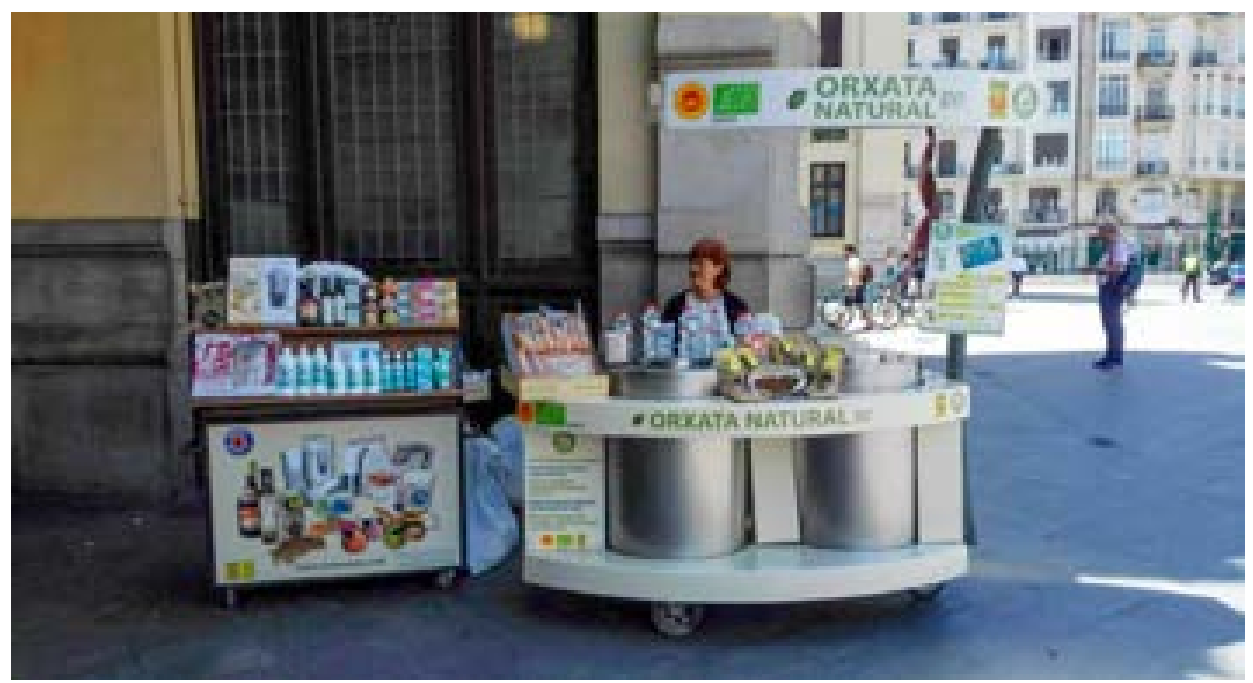

Fuente: elaboración propia

Figura 5. Ejemplo de puesto para el consumo de horchata (III). Valencia

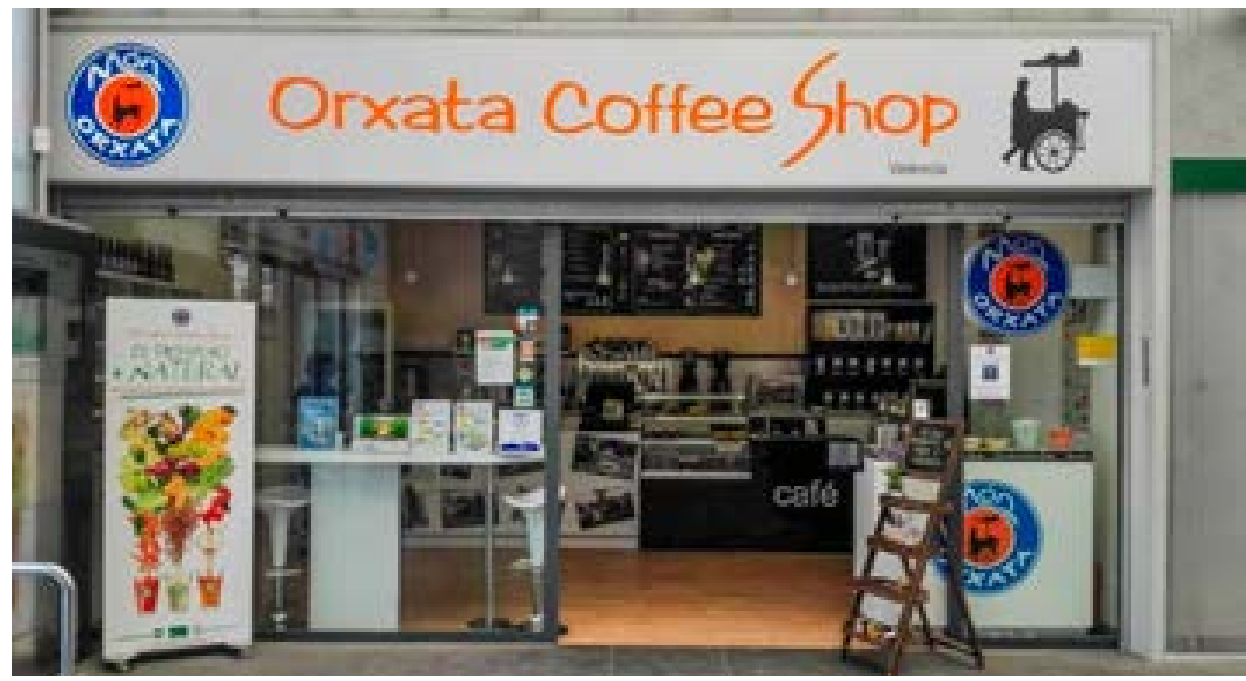

Fuente: elaboración propia 
Figura 6. Venta de horchata y fartons en un supermercado. Valencia

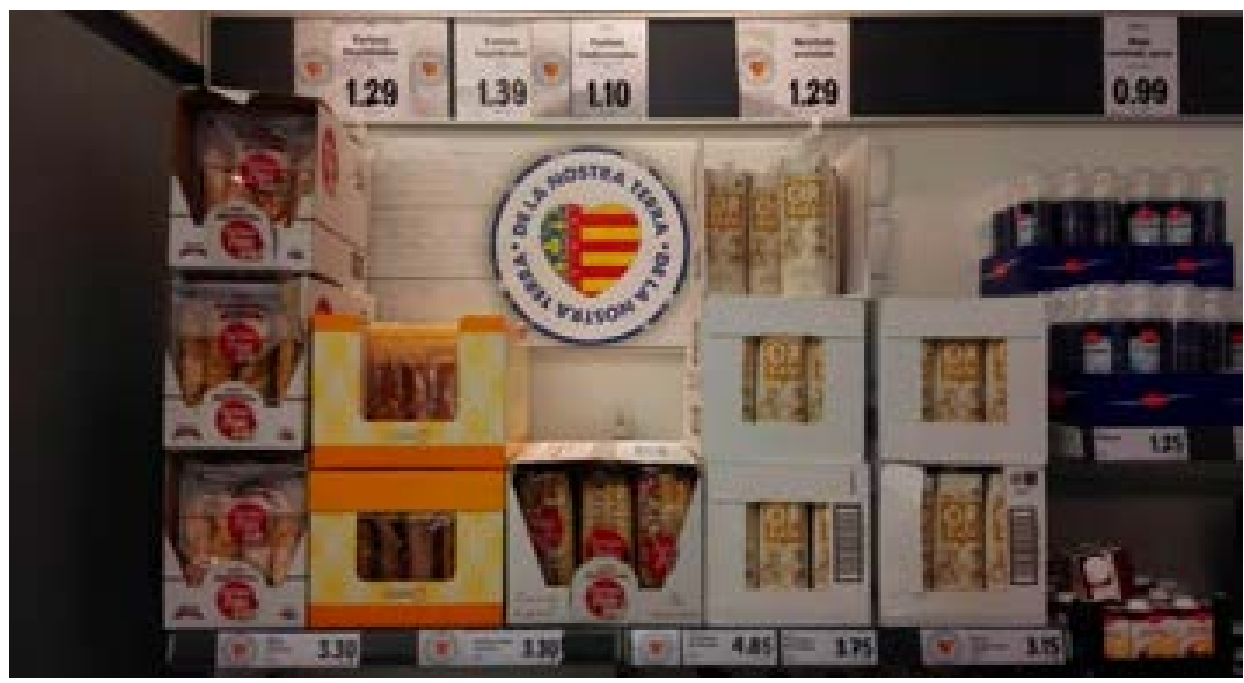

Fuente: elaboración propia

En la misma dirección, y en relación al acompañante de la horchata, de acuerdo con Álvarez (2017), es en la década de los años 60 del siglo XX cuando "un bollo alargado revolucionó la forma de consumirla. Se trata del fartó” (Álvarez, 2017, p. 1). Así pues, el nacimiento de este producto se asocia a la familia Polo:

Dionisio, el mayor de los tres hermanos, y José, un adolescente de apenas 14, solían pasar las horas en el obrador. Preparaban el pan del día siguiente y experimentaban con nuevas creaciones. El fartó surgió de esos ensayos. "Hasta entonces, la horchata se tomaba con rosquilletas o con pan. Se cortaba una vieneta de pan a tiras y se introducía en la bebida granizada. Pero al ser compacto, tarda mucho tiempo en empaparse. Por eso se nos ocurrió la idea de diseñar un producto nuevo, que fuese también alargado y pintamos de azúcar la cara superior", explica José. Los dos hermanos tomaron como base la masa del panquemao e introdujeron algunas modificaciones. [...] Esta innovación sedujo de primeras a las horchaterías que operaban entonces en Alboraya, aunque pronto comenzaron a distribuirse a otros locales de Valencia. La popularidad que alcanzaron los bollos de Polo se extendió como la pólvora. De hecho, en 1973 ya se comercializaban por toda la región gracias a la colaboración de otras ramas familiares. El interés de 
los supermercados por este producto supuso un punto de inflexión. Impulsó el consumo en el hogar y a partir de los 90 abrió de par en par las puertas de otros territorios (Álvarez, 2017, p. 1).

De este modo, los fartons son un producto con unas referencias históricas más recientes. Como una forma de expresión de la cultura gastronómica y las costumbres alimentarias y culinarias valencianas, se puede afirmar que los fartons aparecen íntimamente ligados a la horchata. Fartons Polo (2016) lo describe de la siguiente manera, destacando el nacimiento de los fartons en el año 1960:

En la época del desarrollismo, la familia Polo decidió trasladarse a Alboraya [donde] adquirió un horno en la plaza del pueblo y tras varias pruebas, dieron con un producto perfecto para acompañar a la horchata. Se trataba de un bollo hecho con masa de pan quemao, alargado para ser introducido en el vaso y muy esponjoso para que absorbiera mejor la horchata. Le añadieron la pintura para hacerlo más sabroso y este fue el inicio de lo que hoy conocemos como los Fartons (Fartons Polo, 2016, p. 1).

Más tarde, el conocimiento, la distribución, y la aceptación de los fartons se iba a incrementar, no solo en la región valenciana, sino también en el exterior, tal y como se veía anteriormente. A continuación, a partir de la década de los años 70 del siglo XX,

Dado el éxito alcanzado en Valencia, inmediatamente comenzaron a distribuir los fartons por toda la Comunidad Valenciana y al resto de España, donde ha tenido una gran acogida. A esto está contribuyendo, por ejemplo, el turismo de interior que llega a la Costa Valenciana y que, una vez ha probado la horchata acompañada por tan singular producto, rara es la ocasión en que no quiera repetir y lo busque en su lugar de residencia (Fartons Polo, 2016, p. 1).

De la suma pues de la horchata y los fartons nace un producto que aglutina dos tradiciones identitarias de las tierras valencianas. Como se mencionaba unas líneas más arriba, ambas cuentan con abundantes referencias a lo largo de las calles de Valencia, lo cual se refleja en un uso que incluye tanto a locales como visitantes.

No obstante, Costa (2016) menciona que aún hoy en día para muchos "la horchata se mantiene como una vieja desconocida, como un tesoro por descubrir" (p. $\mathrm{xx}$ ) y lo destaca como un patrimonio culinario y gastronómico no explotado. Aunque su potencial turístico es evidente, afirma que aún son pocos los turistas que lo conocen (Costa, 2016). Como producto tradicional, de base territorial, y elaborado de forma artesanal, sus propiedades para ser un reclamo gastronómico 
son evidentes, y se pueden observar en iniciativas como la Ruta de la Chufa o su papel central en les Falles de València. El consumo de la horchata, además, acompañado de los fartons, resulta en una combinación con otro producto local, artesano, que nace de la tradición vinculada a la panadería y la pastelería, y que logra encontrar en la elaboración -y el consumo- de los fartons el acompañante perfecto de la horchata.

\section{Conclusiones}

La horchata y los fartons son dos señas de identidad de la cultura y la gastronomía valencianas. Sin ir más lejos, la presencia protagonista de la horchata en las Falles, con la repercusión mediática que esto significa, abre también la puerta al conocimiento y a la comunicación de este producto, que se encuentra profundamente presente en la geografía cultural y gastronómica valenciana. Además de los ejemplos escritos y fotográficos observados en la sección anterior, hay otras iniciativas que se suman a la integración de estos productos en los circuitos y paisajes turísticos de Valencia, como por ejemplo la Ruta de la Chufa. Esta ruta recorre las zonas de cultivo de la chufa y sus espacios de producción tradicionales, lo cual permite la descubierta de materiales y construcciones asociados (Consell Regulador D.O. Xufa De València, 2018).

Son también son ejemplos otras actividades que organiza el consejo regulador, como la participación en ferias y exposiciones o las degustaciones y showcookings. Todas estas acciones son proyecciones de la localidad frente a la globalización de la gastronomía actual. Aunque este conflicto no se desarrolla en este trabajo, sí que puede ser un elemento de discusión futura en el momento de realizar estudios empíricos sobre la proyección de la imagen turística de la región.

Si bien entre las limitaciones de este artículo está su carácter principalmente descriptivo, sus implicaciones tanto teóricas como prácticas pueden llevar a varias oportunidades de investigación. En relación con la aportación teórica, este artículo puede servir a estudios posteriores que desarrollen narrativas asociadas a estos productos -tanto desde el punto de vista de la oferta como de la demanda, local, nacional e internacional-.

En la práctica se observan varias implicaciones principalmente para los puntos de venta y distribución, donde se abren posibilidades de desarrollo del producto y de su contexto experiencial. Por ejemplo, aunque la receta original de la horchata se sigue manteniendo en la actualidad -a base de chufas y agua y azúcar-, ya se están realizando otros productos específicos a base de chufas o revalorizándose su uso en los restaurantes en general y los de alta cocina en particular. 
En la misma dirección, la innovación también llega a las formas de poner los productos a disposición de los consumidores, ya sea a través de muestras de street art (Figura 7) o street food (Figura 8), ambos conceptos actuales dentro de la comprensión de las relaciones entre gastronomía, territorio y turismo. En el marco de las sinergias entre alimentación, cocina tradicional, gastronomía y práctica turística, estudios sobre la temática podrían profundizar en las propiedades nutricionales de la horchata y el fartó, el rol que desempeñan en la dieta mediterránea, o la importancia de la DOP para la visibilidad de la producción y la motivación para el consumo.

De este modo, tampoco hay que olvidar que tanto la horchata como los fartons, si bien representan la identidad valenciana, no son los únicos productos gastronómicos que ayudan a construir la imagen culinaria de esta región. El estudio de la composición integral del paisaje gastronómico valenciano puede ser otra línea de investigación, aproximada desde disciplinas como por ejemplo la antropología o el marketing, tanto desde la perspectiva de los locales como de los turistas.

Figura 7. El consumo simbólico de la horchata como parte del street art. Valencia

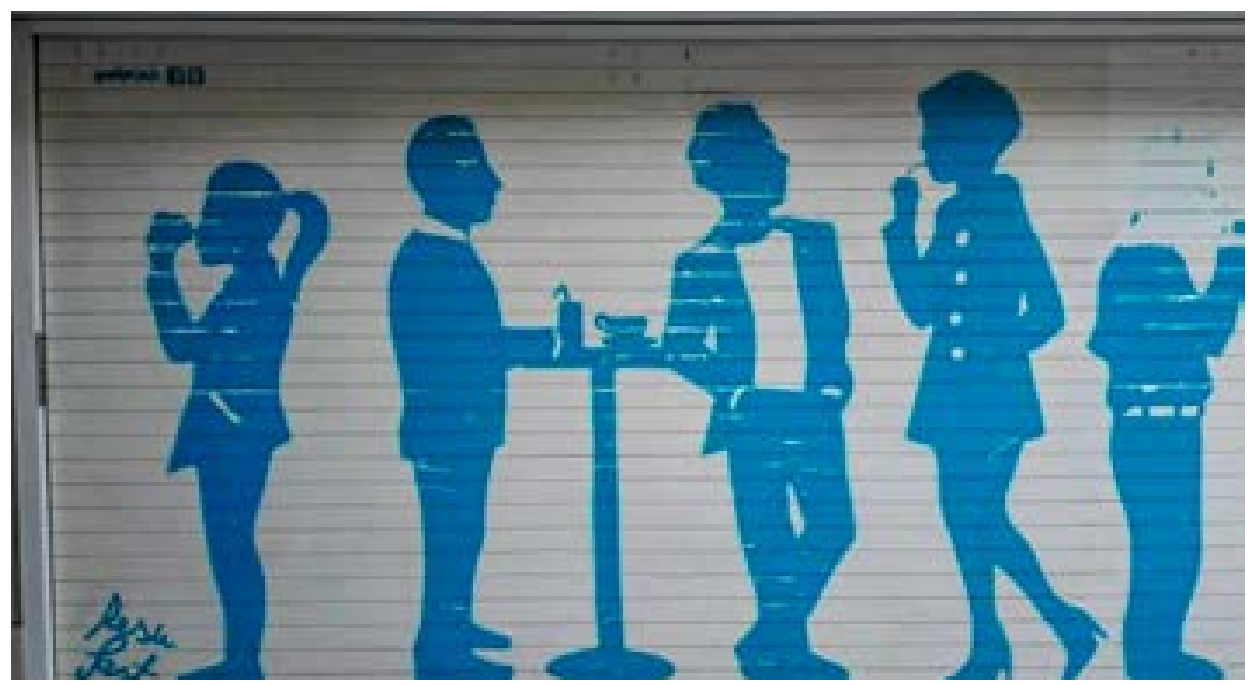

Fuente: elaboración propia 
Figura 8. La oferta tradicional de la horchata con fartons a través del street food. Valencia

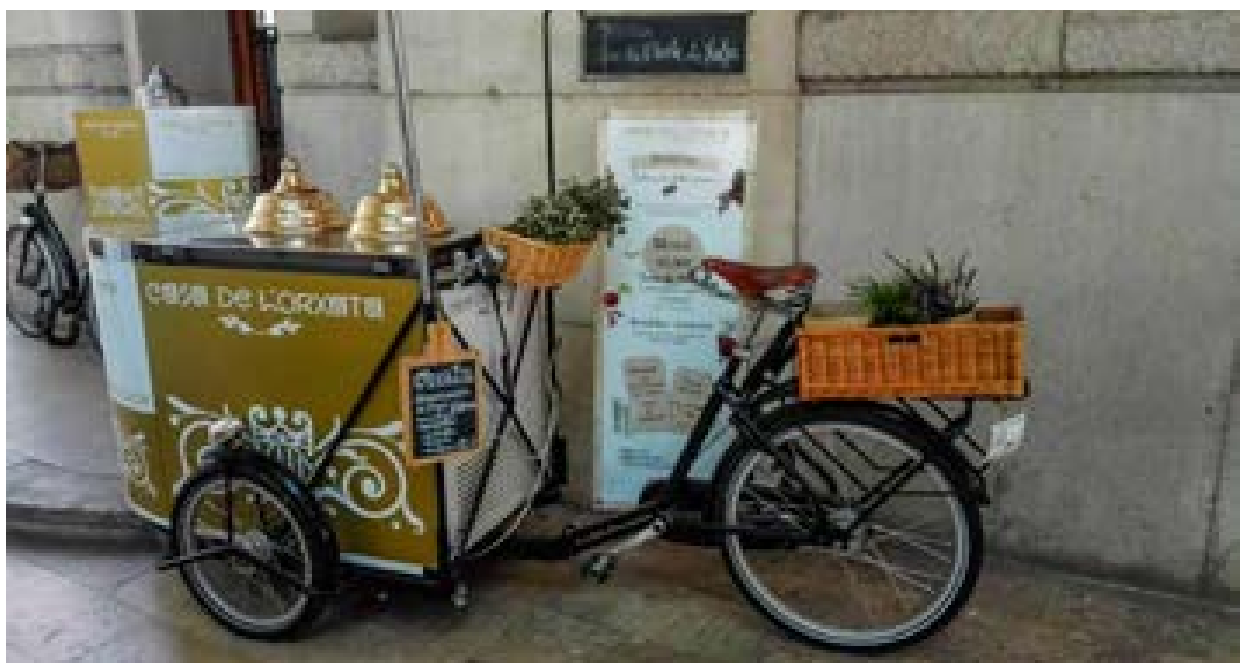

Fuente: elaboración propia

\section{Referencias}

Adema, P. (2006). Festive Foodscapes: Iconizing Food and the Shaping of Identity and Place. Texas, Estados Unidos de Norteamérica: The University of Texas.

Álvarez, F. (20 de agosto de 2017). El panadero que revolucionó la forma de consumir horchata. El Mundo, 1-2. http://www.elmundo.es/comunidad-valenciana/2017/08/20/59992916468aebbe238b45f5.html [Consultado el 3 Mayo de 2018].

Antrop, M. (2005). Why Landscapes of the Past are Important for the Future? Landscape and Urban Planning, 70, 21-34.

https://www.sciencedirect.com/science/article/pii/So16920460300207X [Consultado el 3 Mayo de 2018].

Belisle, F. J. (1983). Tourism and Food Production in the Caribbean. Annals of Tourism Research, 10, 497-513. https://www.sciencedirect.com/science/ article/abs/pii/0160738383900051 [Consultado el 13 Junio de 2018 ]. 
Berno, T. (2017). Building the New Zealand Advantage: Putting the 'Culture' Back Into Agriculture. In Massey, C. (Ed.). The New Zealand Land and Food Annual (pp. 181-192). Palmerston North, Nueva Zelanda: Massey University Press.

Bessière, J. (1998). Local Development and Heritage: Traditional Food and Cuisine as Tourist Attractions in Rural Areas. Sociologia Ruralis, 38(1), 2134. https://onlinelibrary.wiley.com/doi/epdf/10.1111/1467-9523.00061 [Consultado el 13 de Junio de 2018].

Cànoves, G.; Villarino, M. (2000). Turismo en espacio rural en España: Actrices e imaginario colectivo. Documents d'Anàlisi Geogràfica, 37, 51-77. https:// ddd.uab.cat/record/1298 [Consultado el 6 de Mayo de 2018].

Cohen, E.; Avieli, N. (2004). Food in Tourism: Attraction and Impediment. Annals of Tourism Research, 31(4), 755-778. https://www.sciencedirect.com/scien$\mathrm{ce} / \mathrm{article} / \mathrm{abs} / \mathrm{pii} / \mathrm{So} 160738304000623$ [Consultado el 13 Junio de 2018].

Consell Regulador D. O. (2018). Xufa De València. http://va.chufadevalencia. org/ver/244/Hist\%C3ria.html [Consultado el 6 de Mayo de 2018].

Costa, N. (2016). Orxata i fartons, dolços a prova de turistes. Diari Ara, 1-4. https://www.ara.cat/estils_i_gent/Orxata-fartons-turistes-dolcos_O_1634836517.html [Consultado el 3 de Mayo de 2018].

Diari Oficial de la Comunitat Valenciana (2010). Orden 17/2010, de 18 de mayo, de la Conselleria de Agricultura, Pesca y Alimentación, por la que se aprueba el texto del reglamento de la Denominación de Origen Protegida Chufa de Valencia y su consejo regulador. http://www.dogv.gva.es/portal/ficha_disposicion_pc.jsp? sig $=005850 / 2010 \& \mathrm{~L}=1$ [Consultado el 13 de Junio de 2018].

Díaz, I.; Llurdés, J. C. (2013). Reflexiones sobre el turismo de proximidad como una estrategia para el desarrollo local. Cuadernos de Turismo, 32, 65-88. https:// core.ac.uk/download/pdf/25651073.pdf [Consultado el 5 de Mayo de 2018].

Duhart, F.; Medina, F. X. (2008). Els espais socials de la paella: antropología d'un plat camaleònic. Revista d'Etnologia de Catalunya, 32, 88-111. https:// www.raco.cat/index.php/RevistaEtnologia/article/view/81655/106249 [Consultado el 5 de Mayo de 2018].

Fartons Polo (2016). Productos fartons Or, Xata. Valencia, España. http://www. fartonspolo.com/ [Consultado el 5 de Mayo de 2018]. 
Fischler,C.(1988).Food,Self and Identity.SocialScienceInformation, 27(2),275-292. https://journals.sagepub.com/doi/abs/10.1177/053901888027002005 [Consultado el 5 Mayo de 2018].

Fusté-Forné, F. (2015). El turisme gastronòmic: Autenticitat i desenvolupament local en zones rurals. Documents d'Anàlisi Geogràfica, 61(2), 289-304. https://dag.revista.uab.es/article/view/v61-n2-fuste [Consultado el 13 de Junio de 2018].

Fusté-Forné, F. (2016a). El queso como recurso turístico para el desarrollo regional: la Vall de Boí como caso de estudio. Pasos. Revista de Turismo y Patrimonio Cultural, 14(1), 243-251. https://www.redalyc.org/jatsRepo/881/88143642017/index.html [Consultado el 13 de Junio de 2018].

Fusté-Forné, F. (2016b). Los paisajes de la cultura: la gastronomía y el patrimonio culinario. Dixit, 24, 4-16. http://www.scielo.edu.uy/pdf/dix/v24n1/ v24n1a01.pdf [Consultado el 5 de Mayo de 2018].

Fusté-Forné, F. (2019). Seasonality in Food Tourism: Wild Foods in Peripheral Areas. Tourism Geographies, 1-21. https://www.tandfonline.com/doi/full/ 10.1080/14616688.2018.1558453 [Consultado el 1 de Octubre de 2019].

Guerra, I. R.; Moreno, V. M.; López, M. M. (2014). El oleoturismo como atractivo turístico en el medio rural español. Papers de Turisme, 49, 89-103. http:// www.papersdeturisme.gva.es/ojs/index.php/Papers/article/view/170 [Consultado el 5 de Mayo de 2018].

Hall, C. M.; Sharples, L. (2003). The Consumption of Experiences or the Experience of Consumption? An introduction to the tourism of taste. En Hall, C.M., Sharples, L., Mitchell, R., Macionis, N., Cambourne, B. (Eds.). Food Tourism Around the World: Development, Management and Markets (pp. 1-24). Oxford, Inglaterra: Elsevier.

Hall, C. M., Sharples, L.; Cambourne, B.; Macionis, N. (2009). Wine Tourism Around the World. Londres, Inglaterra: Routledge.

Hernández-Rojas, R. D.; Dancausa-Millán, M. G. (2018). Turismo Gastronómico. La gastronomía tradicional de Córdoba (España). Estudios y perspectivas en turismo, 27(2), 413-430. http://www.scielo.org.ar/pdf/eypt/v27n2/ v27n2a12.pdf [Consultado el 5 de Mayo de 2018]. 
Hillel, D.; Belhassen, Y.; Shani, A. (2013). What Makes a Gastronomic Destination Attractive? Evidence from the Israeli Negev. Tourism Management, 36, 200-209. http://www.bgu.ac.il/ yanivbel/pdf\%20files/Gastronomic\%20 tourism\%20in\%20the\%20Negev.pdf [Consultado el 13 de Junio de 2018].

Hjalager, A. M.; Richards, G. (2002). Tourism and Gastronomy. Nueva York, Estados Unidos de Norteamérica: Routledge.

Hughes, G. (1995). Authenticity in Tourism. Annals of Tourism Research, 22(4), 781-803. https://www.sciencedirect.com/science/article/abs/pii/016073839500020X [Consultado el 3 de Mayo de 2018].

Kim, S.; Ellis, A. (2015). Noodle Production and Consumption: From Agriculture to Food Tourism in Japan. Tourism Geographies, 17(1), 151-167. https:// www.tandfonline.com/doi/abs/10.1080/14616688.2014.978812 [Consultado el 3 de Mayo de 2018].

Korstanje, M. E. (2010). Las formas elementales de la hospitalidad. Revista Brasileira de Pesquisa em turismo, 4(2), 86-111. https://rbtur.org.br/rbtur/article/view/325/347 [Consultado el 13 de Junio de 2018].

Leal-Londoño, M. P.; Vázquez-Medina, J. A.; Medina, F. X. (2018). Gastronomy and Tourism: Blending Local Essence and Global Logic. The Case of Basque Taverns in Barcelona's El Poble Sec Neighbourhood. Anthropology of Food, 13, 1-23. https://journals.openedition.org/aof/8500 [Consultado el 13 de Junio de 2018].

López-Guzmán, T., García, J. R.; Rodríguez, Á. V. (2013). Revisión de la literatura científica sobre enoturismo en España. Cuadernos de Turismo, 32, 171-188. https://revistas.um.es/turismo/article/view/177511/149241 [Consultado el 3 de Mayo de 2018].

Lund, K. A. (2015). Magic Mussels: Ingredients for Improvising a Tourism Destination. Journal of Gastronomy and Tourism, 1, 19-31. https://www.ingentaconnect.com/content/cog/gat/2015/00000001/00000001/art00003;jsessionid=qkpftm6rghhj.x-ic-live-03 [Consultado el 3 de Mayo de 2018].

Maroto, J. V. (1998). Historia de la Agronomía. Madrid, España: Mundi Prensa. 
Organización Mundial del Turismo (2001). Apuntes de Metodología de la Investigación en Turismo. Madrid, España: Organización Mundial del Turismo.

Pardinas, F. (1969). Metodología y técnicas de investigación en ciències sociales. México, D.F.: Siglo XXI Editores.

Pascual, B.; Maroto, J. V. (1984). Estudios agronómicos realizados en el cultivo de la chufa (Cyperus esculentus L.). Valencia, España: Diputación Provincial de Valencia.

Pons, P. (2018). El templo de la horchata en Alboraya. Guía Hedonista, 1-2. https://valenciaplaza.com/daniel-el-hombre-que-mojo-el-farton-en-lahorchata-por-primera-vez [Consultado el 5 de Mayo de 2018].

Serrallach, J. (1927). Die wurzelknolle von Cyperus esculentus L. Frankfurt, Alemania: Universität Frankfurt am Main.

Sims, R. (2009). Food, Place and Authenticity: Local Food and the Sustainable Tourism Experience. Journal of Sustainable Tourism, 17(3), 321-336. https://www.tandfonline.com/doi/abs/10.1080/09669580802359293 [Consultado Mayo 5 de 2018].

Sirvent Barcelona (2018). Sirvent: más allá de la horchata. https://www.gastronomiaalternativa.com/ga-14_95-sirvent-mas-alla-de-la-horchata.html [Consultado el 3 de Mayo de 2018].

Smith, S. (2015). A Sense of Place: Place, Culture and Tourism. Tourism Recreation Research, 4O(2), 220-233. https://www.tandfonline.com/doi/abs/10. 1080/02508281.2015.1049814 [Consultado el 3 de Mayo de 2018].

Somekh, B.; Lewin, C. (2005). Research Methods in the Social Sciences. Londres, Inglaterra: SAGE Publications.

Tellström, R., Gustafsson, I.; Mossberg, L. (2006). Consuming Heritage: The Use of Local Food Culture in Branding. Place Branding, 2(1), 130-143. https://link.springer.com/article/10.1057/palgrave.pb.5990051 [Consultado el 5 de Mayo de 2018].

Tresserras, J. J.; Medina, F. X. (2008). Patrimonio gastronómico y turismo cultural en el Mediterráneo. Barcelona, España: Ibertur. 


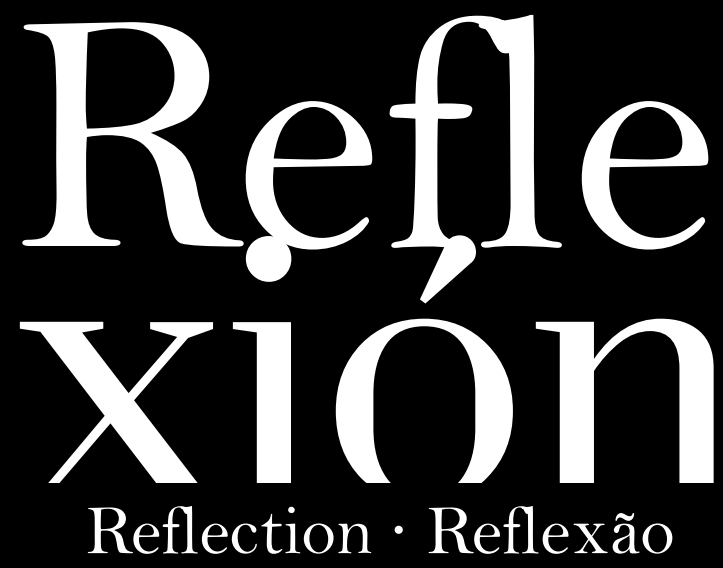


